\title{
Research on Intensive Care Experiences of Patients in Mechanical Ventilation
}

\author{
Jinliang Hu*, Qing Ye, Chanjuan Ji \\ Department of Operating, Binzhou People's Hospital, Binzhou, China
}

Email address:

hujinliang2019@163.com (Jinliang Hu)

${ }^{*}$ Corresponding author

To cite this article:

Jinliang Hu, Qing Ye, Chanjuan Ji. Research on Intensive Care Experiences of Patients in Mechanical Ventilation. American Journal of BioScience. Vol. 7, No. 5, 2019, pp. 88-93. doi: 10.11648/j.ajbio.20190705.12

Received: August 31, 2019; Accepted: October 26, 2019; Published: November 8, 2019

\begin{abstract}
To quantitatively analyse conditions of intensive care experiences and influencing factors of patients. A database was established, it can be seen from the overall experience index that in the SD dimensionality, 46.11\% patients have highly bad experiences and $86.11 \%$ patients have moderate and highly bad experiences in total plus the patients with moderate scores; in ET dimensionality, $32.78 \%$ patients have highly bad experiences and $67.22 \%$ patients have moderate and highly bad experiences in total plus the patients with moderate scores; in TII dimensionality, 73.88\% patients have moderate and highly bad experiences. $\mathrm{L}_{\mathrm{ICU}}$ and $\mathrm{L}_{\mathrm{MV}}$ of patients and overall GEI and various factor scores exhibit a significant positive correlation $(\mathrm{P}<0.05)$ indicating that the longer the time of mechanical ventilation treatment and length of stay in ICU, the more bad experiences the patients will have; patients' APACHE scores and the overall GEI, dimensionality ET, ACF and TII exhibit a positive correlation (P<0.05) indicating the severer the disease is, the more bad experiences on intensive care the patient will have. Mechanical ventilation duration, disease severity degrees, marital status and age are the major factors influencing intensive care experiences of patients in mechanical ventilation.
\end{abstract}

Keywords: Mechanical Ventilation, Intensive Care, Scale

\section{Introduction}

With scientific and technical progress in medical care, as an important respiratory support approach, mechanical ventilation has been increasingly applied in the intensive care thus saving the lives of many critically ill patients. The mechanical ventilation not only serve as one of the important means for anesthetization and emergency treatment but also a common treatment means for respiratory support for critically ill patients. The application range of the mechanical ventilation has been expanding in the medical field [1-2]. A large scale survey on conditions of ICU patients by Mealer et al. indicates that $39 \%$ of the ICU patients received mechanical ventilation in treatment [3]. Therefore, the patients will receive mechanical ventilation in ICU every day. There are a number of studies on mechanical ventilation and extubation whereas these studies focus on discussions on mechanical ventilation strategies and extubation strategies for different patients from the physiological index and technological aspects and seldom focus on subjective experiences of these patients [4-5]. The existing studies on subjective experiences of the ICU patients include not only the patients receiving mechanical ventilation but also the patients receiving other ICU treatment. The mechanical ventilation serves as a common measure in ICU treatment nevertheless there is no quantitative study with the patients receiving mechanical ventilation as the research object [6].

In the study, a standard scale was used to evaluate the intensive care experiences of patients in mechanical ventilation and analyse relevant factors influencing the patients' experience. It is expected to expand the understanding of the intensive care experiences of patients in mechanical ventilation by the medical care personnel and promote holistic nursing of the patients. 


\section{Material and Methods}

\subsection{Material}

The patients who experienced mechanical ventilation treatment and survive in ICU at our hospital from June 2018 to June 2019 were selected as the research objects. Inclusion criteria for the research objects: 1. the length of stay in ICU was more than or equal to $48 \mathrm{~h}$; the patient received mechanical ventilation treatment; 2 . aged more than 18 years. Exclusion criteria for the research objects: 3. patients who had underwent visceral organ transplantation; 4. patients who underwent trachea intubation for more than $24 \mathrm{~h}$ at another hospital; 5. patients with chronic mechanical ventilation dependency; 6. patients with senile dementia before hospitalization; 7. patients with a Chinese communication disorder [7].

The eligible patients were investigated. Based on the estimation method of multivariable study on sample size, the sample size was determined with 5-times of the number of variables. The number of scale items used in the study was 23 at most and the sample size determined primarily was 150 200.

\subsection{Survey of General Conditions}

Based on the demand in the study, the survey was to be designed by the researcher. The content included the age, gender, occupation, marital status, educational level, payment mode, length of stay in ICU ( $\left.\mathrm{T}_{\mathrm{ICU}}\right)$, main diagnosis, previous health conditions, mechanical ventilation duration ( $\left(\mathrm{T}_{\mathrm{MV}}\right)$ etc. of the research objects.

\subsection{Acute Physiology and Chronic Health Evaluation (APACHE)}

APACHE is a tool used to evaluate the conditions of critically ill patients and predict mortality. The most clinically and widely used APACHE II scoring system is adopted in the study to evaluate the conditions of patients in mechanical ventilation being admitted to ICU. The APACHE II scoring system comprises acute physiology score, patient age score, and the chronic health condition score. The data collected is the worst values within 24 hours after the patient was admitted to ICU or rescued and a higher score indicates a server condition. A number of clinical studies have shown that it can predict in-hospital mortality and conditions of the patients timely, accurately and subjectively [8].

\subsection{Intensive Care Experience Scale (ICES)}

The scale includes 23 items reflecting the five dimensionalities of the patients' intensive care experience: awareness of surrounding (AS), experience technique (ET), altered cognition and feeling (ACF), inter-personal support demands (SD), and treatment and illness information (TII), which have high reliability and validity. Two answering modes are used in the scale: consent mode and frequency mode. The consent mode contains 11 items and the 5-level scoring method is used: 1-very agree, 2-agree, 3-uncertain, 4-disagree, 5-very disagree The frequency mode contains 12 items and the 5-level scoring method is also used: 1-all time, 2-most time, 3-sometimes, 4-seldom, 5-never To avoid any response set of the research objects tested, the positive and negative scoring is used. The number of items for positive scoring is 5 and the number of items for negative scoring is 18 . A higher score indicates more bad experiences the patient has during intensive care. The General Experience Index (GEI) (total scores of all items/total number of items) and factor scores (total score of each dimensionality / number of items of the dimensionality) were evaluated for easy comparison due to the fact that the number of items of each dimensionality was unequal. The items of more than 2 points were rated as bad experience, the items with score ranging from 2-2.49 were rated as slightly bad experience, the items with score ranging from 2.5-3.49 were rated as moderately bad experience, and the items with score above 3.5 were rated as severely bad experience [9].

\subsection{Data Collection Process}

The eligible research objects were contacted first and then invited to participate the study after discharged from ICU. The patients were informed of the research objective, benefits and dangers of involvement in the study, voluntary participation principle, and the brief process of the study. The questionnaires were distributed to the patients after the informed consent from the patients was obtained and before the patients were discharged from the hospital and 3 days after released from ICU. The patients would fill in the questionnaires based on their memory on ICU intensive care experiences. Principally, the inquirer was required to explain to the patients face to face using the uniform instruction and the scale was to be filled in by the patients. The inquirer would ask questions if the patient was not able to fill the scale due to conditions or physical weakness. The inquirer would fill in the scale objectively and faithfully based on the answers of the patient. The questionnaires were to be collected timely after completion.

\subsection{Data Sorting}

The questionnaires collected were numbered uniformly and the items checked for carefulness and completion. 1. The questionnaires where the answers for all items shared the same option were rejected; 2 . The questionnaires with three or more items unanswered were rejected. 3. The omitted items of the questionnaires with one or two items unanswered would be supplemented as per the average value of the items.

\subsection{Statistic Analysis of Data}

A database was established for all data of the valid questionnaires and the SPSS 13.0 software was used for analysis. The descriptive analysis was used to describe the general data conditions of the patients, conditions of intensive care experience of the patients; the independent sample $t$ test and one-way analysis of variance were used to analyse the relationship between the classified variables of gender, 
educational level and expense source and the intensive care experience of the patients; the Pearson related analysis was used to analyse the relationship between the numerical variables of the mechanical ventilation time, length of stay in ICU, APACHE scores etc. of the patients and the intensive care experience of the patients; with the total score of intensive care experience and scores of various dimensionalities as the dependent variables and the above-mentioned various demographic factors, diseases, treatment factors etc. with a significant difference to the intensive care experience, multiple stepwise regression was performed to further assess the internal relationship between various demographic factors, diseases, treatment factors etc. and intensive care experience of the patients.

\section{Results}

\subsection{Research Objects and Data Collection}

During data collection, 714 patients aged over 18 years in ICU at our hospital received invasive mechanical ventilation treatment. 92 of them died during hospitalization (12.89\%); among the remaining 622 patients, 233 (32.63\%) were not interviewed due to severe conditions or unconsciousness, or lack of normal responses to people, location, status etc. 43 patients $(6.02 \%)$ could not remember that they received respirator treatment or stayed in an ICU. 162 patients $(22.79 \%)$ or their family members were unwilling to participate in the study. The final number of participant patients was 184 .

Relevant questionnaires were distributed to the 184 patients meeting the inclusion criteria and agreeing to participate in the study for filling in as required. The number of questionnaires collected was 184.4 of them had three or more unanswered items and were rejected as unacceptable; 2 of them had one or two omitted items and such omitted items were supplemented in accordance with the average value of the items. Therefore, there are 180 valid questionnaires and the valid collection rate is $97.83 \%$.

\subsection{General Data of Research Objects}

See Table 1 for the demographic and disease characteristics of the 180 patients with valid questionnaires returned.

Table 1. Demographic and Disease Characteristics of the Research Objects.

\begin{tabular}{|c|c|c|c|}
\hline Characteristic & & Number/Range & Percentage $/ \pm$ s \\
\hline \multirow{2}{*}{ Gender } & Male & 106 & $58.89 \%$ \\
\hline & Female & 74 & $41.11 \%$ \\
\hline \multirow[t]{2}{*}{ Age } & & $18-80$ & $45.72 \pm 18.16$ \\
\hline & Primary School and Lower & 48 & $26.67 \%$ \\
\hline \multirow[t]{2}{*}{ Educational Level } & Junior and Senior High School & 70 & $38.89 \%$ \\
\hline & College and Higher & 62 & $34.44 \%$ \\
\hline Marital Status & Married & 152 & $84.44 \%$ \\
\hline \multirow{2}{*}{ Cost Source } & Medical Insurance & 131 & $72.78 \%$ \\
\hline & Miscellaneous & 49 & $27.22 \%$ \\
\hline APACHE Score & & $10-40$ & $23.74 \pm 13.82$ \\
\hline Length of Stay in ICU (hours) & & $72-720$ & $424.9 \pm 168.3$ \\
\hline Mechanical Ventilation Time (hours) & & $12-480$ & $261.5 \pm 118.4$ \\
\hline \multirow{2}{*}{ Cause for Invasive Mechanical Ventilation } & Respiratory Failure & 65 & $36.11 \%$ \\
\hline & Post Cardiopulmonary Resuscitation & 22 & $12.22 \%$ \\
\hline
\end{tabular}

\subsection{Descriptive Statistics of Intensive Care Experiences of the Patients}

The intensive care experiences of the patients are reflected in five dimensionalities, i.e. general experience index and awareness of surrounding (AS), experience technique (ET), altered cognition and feeling (ACF), inter-personal support demands (SD), treatment and illness information (TII). It can be seen from the general experience index that in $\mathrm{SD}, 46.11 \%$ patients have high bad experiences and $86.11 \%$ patients have moderately and highly bad experiences plus patients with moderate scores in total; in ET, $32.78 \%$ patients have highly bad experiences and $67.22 \%$ patients have moderately and highly bad experiences plus patients with moderate scores in total; in TII, 73.88\% patients have highly bad experiences. The overall distribution conditions are shown in Table 2.

Table 2. Overall Conditions of Intensive Care Experiences of Patients in Mechanical Ventilation.

\begin{tabular}{lllll}
\hline Dimensionality & \pm s & Fair $(\mathbf{n} / \%)$ & Slightly Bad (n/\%) & Moderately Bad (n/\%) \\
\hline AS & $3.07 \pm 0.28$ & $(31 / 17.22)$ & $(55 / 30.55)$ & $(87 / 48.33)$ \\
ET & $3.32 \pm 0.36$ & $(14 / 7.78)$ & $(45 / 25.00)$ & $(62 / 34.44)$ \\
ACF & $2.08 \pm 0.37$ & $(115 / 63.89)$ & $(42 / 23.33)$ & $(14 / 7.78)$ \\
SD & $3.76 \pm 0.29$ & $(6 / 3.33)$ & $(19 / 10.56)$ & $(72 / 40.00)$ \\
TII & $3.25 \pm 0.33$ & $(9 / 5.00)$ & $(38 / 21.11)$ & $(107 / 59.44)$ \\
GEI & $3.11 \pm 0.32$ & $(17 / 9.44)$ & $(43 / 23.89)$ & $(112 / 62.22)$ \\
\hline
\end{tabular}




\subsection{Effect of Sex Factors on Intensive Care Experiences of Patients}

The independent sample $\mathrm{t}$ test was applied to analyse differences in various aspects of the intensive care experiences of patients of different genders. The result has shown no statistical significance $(\mathrm{P}>0.05)$. The intensive care experiences of patients of different genders have no significant difference. See Table 3.

Table 3. Comparison of Intensive Care Experiences of Patients of Different Genders.

\begin{tabular}{llll}
\hline Dimensionality & Male $(\mathbf{x} \pm \mathbf{s})$ & Female $(\mathbf{x} \pm \mathbf{s})$ & P Value \\
\hline AS & $3.14 \pm 0.31$ & $3.10 \pm 0.38$ & 0.668 \\
ET & $3.53 \pm 0.44$ & $3.42 \pm 0.33$ & 0.292 \\
ACF & $2.07 \pm 0.38$ & $2.18 \pm 0.34$ & 0.287 \\
SD & $3.57 \pm 0.41$ & $3.81 \pm 0.42$ & 0.183 \\
TII & $3.37 \pm 0.36$ & $3.44 \pm 0.40$ & 0.381 \\
GEI & $3.08 \pm 0.37$ & $3.13 \pm 0.35$ & 0.416 \\
\hline
\end{tabular}

\subsection{Effect of Age Factors on Intensive Care Experiences of Patients}

The patients were divided into three groups based on age, $\leq 40,40-60$ and $\geq 60$. An one-way analysis of variance was conducted for intensive care experiences of the patients at different ages in ICU undergoing the five dimensionalities and the overall conditions. The result has shown that there is a significant difference in GEI scores among various age groups $(\mathrm{P}<0.05)$. The younger the age was, the higher the GEI score would be. The scores of the age group of 40-60 years in ET dimensionality are significantly higher than that of the remaining two groups $(\mathrm{P}<0.05)$. GEI, AS and $\mathrm{SD}$ of the $>60$ group in intensive care are significantly lower than that of other groups $(\mathrm{P}<0.05)$. There is no difference in the remaining dimensionalities among patients at different ages. See Table 4.

Table 4. Comparison of Intensive Care Experiences of Patients at Different Ages.

\begin{tabular}{lllll}
\hline Dimensionality & $\mathbf{5 4 0}(\mathbf{n}=\mathbf{3 5})$ & $\mathbf{4 0 - 6 0}(\mathbf{n}=\mathbf{8 6})$ & $\mathbf{2 6 0}(\mathbf{n}=\mathbf{5 9})$ & P Value \\
\hline AS & $3.25 \pm 0.43$ & $2.93 \pm 0.30$ & $2.37 \pm 0.42$ & $0.009^{*}$ \\
ET & $3.07 \pm 0.44$ & $3.78 \pm 0.41$ & $3.14 \pm 0.37$ & $0.026^{*}$ \\
ACF & $2.24 \pm 0.51$ & $2.13 \pm 0.38$ & $2.05 \pm 0.36$ & 0.542 \\
SD & $3.83 \pm 0.37$ & $3.61 \pm 0.33$ & $2.98 \pm 0.40$ & $0.011^{*}$ \\
TII & $3.25 \pm 0.42$ & $3.36 \pm 0.36$ & $3.09 \pm 0.32$ & 0.282 \\
GEI & $3.40 \pm 0.48$ & $3.08 \pm 0.41$ & $2.81 \pm 0.35$ & $0.032^{*}$ \\
\hline
\end{tabular}

Legend, $* \mathrm{P}<0.05, \mathrm{vs} \geq 60$.

\subsection{Effect of Disease Treatment Factors on Intensive Care Experiences of Patients}

Difference in types of ICUs of the intensive care experiences of the patients: A one-way analysis of variance was conducted for the patients in ICU in five dimensionalities and the overall conditions. The result has shown that the overall GEI score of emergent ICU patients is significantly higher than that of the ICU patients of other types $(\mathrm{P}<0.05)$. There is a significant difference among patients in different ICUs in AS, ET, ACF and TII $(\mathrm{P}<0.05)$ and no difference in SD $(\mathrm{P}>0.05)$. See Table 5.

Table 5. Comparison of Intensive Care Experiences of Patients in ICUs of Different Types.

\begin{tabular}{|c|c|c|c|c|}
\hline Dimensionality & Internal Medicine ICU $(n=72)$ & Surgical ICU $(n=55)$ & Emergent ICU $(n=53)$ & P Value \\
\hline AS & $1.92 \pm 0.38$ & $2.54 \pm 0.45$ & $2.92 \pm 0.36$ & $0.005^{*}$ \\
\hline ET & $2.85 \pm 0.37$ & $2.16 \pm 0.33$ & $3.38 \pm 0.43$ & $0.012 *$ \\
\hline $\mathrm{ACF}$ & $2.02 \pm 0.42$ & $2.37 \pm 0.44$ & $2.59 \pm 0.40$ & $0.016^{*}$ \\
\hline TII & $3.44 \pm 0.36$ & $3.16 \pm 0.42$ & $2.73 \pm 0.48$ & $0.013^{*}$ \\
\hline GEI & $2.71 \pm 0.38$ & $2.84 \pm 0.37$ & $3.51 \pm 0.42$ & $0.008 *$ \\
\hline
\end{tabular}

Legend, ${ }^{*} \mathrm{P}<0.05$, vs emergent ICU.

\subsection{Correlation Analysis on the Influencing Factors of Intensive Care Experiences}

The Pearson correlation analysis result has shown that $\mathrm{L}_{\mathrm{ICU}}$ and $\mathrm{L}_{\mathrm{MV}}$ of patients and overall GEI and various factor scores exhibit a significant positive correlation $(\mathrm{P}<0.05)$ indicating that the longer the time of mechanical ventilation treatment and length of stay in ICU, the more bad experiences the patients will have; patients' APACHE scores and the overall GEI, dimensionality ET, ACF and TII exhibit a positive correlation $(\mathrm{P}<0.05)$ indicating the severer the disease is, the more bad experiences on intensive care the patient will have. The experiences are concentratedly reflected in ET, ACF and TII. See Table 6 for the results.

Table 6. Correlation Coefficient of $L_{I C U}, L_{M V}$ and APACHE Scores and General GEI and Various Factors of the Patients.

\begin{tabular}{llll}
\hline Dimensionality & Correlation Coefficient with $\mathbf{L}_{\mathbf{I C U}}$ & Correlation Coefficient with $\mathbf{L}_{\mathbf{M v}}$ & Correlation Coefficient with APACHE \\
\hline AS & $0.143^{*}$ & $0.127^{*}$ & 0.048 \\
ET & $0.492^{*}$ & $0.536^{*}$ & $0.393^{*}$ \\
ACF & $0.207^{*}$ & $0.214^{*}$ & $0.187^{*}$ \\
SD & $0.191^{*}$ & $0.196^{*}$ & 0.061 \\
TII & $0.105^{*}$ & $0.118^{*}$ & $0.114^{*}$ \\
GEI & $0.357^{*}$ & $0.382^{*}$ & $0.276^{*}$ \\
\hline
\end{tabular}

Legend: $* \mathrm{P}<0.05$ indicates the difference has statistical significance. 


\section{Discussion}

During the research, 714 patients in the ICU received invasive mechanical ventilation treatment but only 180 patients participated in the research actually. The main reasons include: 1 . high in-hospital mortality rate of ICU critically ill patients; 2 . the great majority of the patients survived are not able to assist in completing the study due to severe conditions or unconsciousness, or lack of normal responses to the people, location, status etc.; 3 . The patients are not willing to participate and the patients or their family members disagree to participate in the study. It is thus clear that it is difficult to collect data and conduct a study in such a group of population, which is also exactly the primary cause for a small sample size in previous relevant studies [10-11].

Studies have discovered that none of the patients are able to completely remember all experiences during intensive care and approximately $10 \%$ patients are not able to recall their stay in ICU or mechanical ventilation at all. Based on an analysis on the general data the patients, the disease severity levels of such patients without such memories are higher than that of the patients with memories on ICU and mechanical ventilation. A study by Weinert et al. has also shown that the patients of a higher severity level have a poorer memory on ICU [12]. Many foreign studies have revealed that there are many causes for memory loss of the patients staying in ICU, such as disease severity level, mechanical ventilation time, therapeutic medications (including sedative), and the data collection time. The factors above-mentioned may be the causes for the above difference [13-15]. In addition, the research objects in the study do not include the patients with a nervous system disease, which is also a cause for the above difference.

It was discovered in the study that each research object is a unique individual and the intensive care experience differs from that of others. A statistic analysis on the data on such unique individuals indicated that the patients in mechanical ventilation experienced moderate and higher level of intensive care experience, which was consistent with the previous results of researches with westerners as the research objects [16-17]. Baudry has reported cases mental disturbance arising from ICU care and proposed the concept of intensive care syndrome [18]. Some scholars have proposed that the ICU syndrome is a individual mode generated by the patient in ICU. The clinical symptoms can persist for a very long period after the patient is discharged from ICU, which are primarily manifested by mental state changes of the patients in a high stress environment, delirium, clouding of consciousness, insomnia, nightmare, untruthful experiences etc. as well as tension and fear [19-21].

The research analyses the internal relationship between demographic factors, disease and treatment factors etc. and intensive care experiences. The result shows that the main influencing factors for the intensive care experiences of patients in mechanical ventilation include mechanical ventilation duration, disease severity level, marital status, age etc. A longer period of mechanical ventilation indicates a severe disease condition, lack of marital support, younger age of the patient, higher score of the intensive care experience of the patient and more bad experiences. Mechanical ventilation is a major factor resulting in bad experiences for a patient. Existence of mechanical ventilation and tracheal intubation solves the ventilation problem of the patient but brings about substantially bed experiences and psychological fear to the patient. The patients worry that they are unable to respire forever and they may die etc. [22-23]. Marriage and family are important sources of social support. The social support enables people to obtain help from the society or others from the social relation network. The social support is typically considered as potential resources for individuals to deal with stressful events [24-25]. Currently, many studies have shown that the social support is an important intervening variable influencing the result of a stress response. It generally plays a role in mitigating the stress response and exhibits a negative correlation with the physical and psychological response arising from the stress [26-27]. Good social support contributes to physical and psychological health of individuals [28].

\section{Conclusion}

Therefore, the bad experiences with intensive care of the patients lacking of marriage and family support will be severer [29]. Young patients usually have higher requirements and pursue higher living quality. They will produce an intense stress response when they suffer from a serious disease and are admitted to the ICU. Loiselle et al. opined that the effect of ICU experiences on young patients is particularly significant and the research result is consistent with the above [30].

\section{References}

[1] Castro AA. Calil SR. et al. Chest physiotherapy effectiveness to reduce hospitalization and mechanical ventilation length of stay, pulmonary infection rate and mortality in ICU patients $[\mathrm{J}]$. Respir Med, 2019, 107 (1): 68-74.

[2] Anon JM. Gomez-Tello V. et al. Prognosis of elderly patients subjected to mechanical ventilation in the ICU [J]. Med Intensiva, 2019, 37 (3): 149-155.

[3] Mealer M. Lareau SC. Pulmonary processes and mechanical ventilation in the ICU patient [J]. Crit Care Nurs Clin North Am, 2018, 24 (3): 152-3.

[4] Futier E. Chanques G. et al. Influence of opioid choice on mechanical ventilation duration and ICU length of stay $[\mathrm{J}]$. Minerva Anestesiol, 2018, 78 (1): 46-53.

[5] Penuelas O. Frutos-Vivar F. et al. Unplanned extubation in the ICU: a marker of quality assurance of mechanical ventilation [J]. Crit Care, 2011, 15 (2): 128-9.

[6] Fanfulla F. Ceriana P. et al. Sleep disturbances in patients admitted to a step-down unit after ICU discharge: the role of mechanical ventilation [J]. Sleep, 2011, 34 (3): 355-62. 
[7] Yiliaz C. Kelebek Girgin N. et al. The effect of nursing-implemented sedation on the duration of mechanical ventilation in the ICU [J]. Ulus Travma Acil Cerrahi Derg, 2010, 16 (6): 521-6.

[8] Cooke CR. Hotchkin DL. et al. Predictors of time to death after terminal withdrawal of mechanical ventilation in the ICU $[\mathrm{J}]$. Chest, 2010, 138 (2): 289-97.

[9] Cline SD. Schertz RA. et al. Expedited admission of patients decreases duration of mechanical ventilation and shortens ICU stay [J]. Am J Emerg Med, 2009, 27 (7): 843-6.

[10] Bennett J. Protocol-directed sedation did not reduce duration of mechanical ventilation or hospital stay in ICU patients [J]. Evid Based Nurs, 2008, 11 (4): 124-6.

[11] Hewson-Conroy KM. Tierney LT. et al. Assessment and perceptions of intensive care data quality, reporting and use: a survey of ICU directors [J]. Anaesth Intensive Care, 2018, 40 (4): $675-82$

[12] Weinert CR. Sprenkle M. Post-ICU consequences of patient wakefulness and sedative exposure during mechanical ventilation [J]. Intensive Care Med, 2008, 34 (1): 82-90.

[13] Gusmao-Flores D. Figueira Salluh JI. The confusion assessment method for the intensive care unit (CAM-ICU) and intensive care delirium screening checklist (ICDSC) for the diagnosis of delirium: a systematic review and meta-analysis of clinical studies [J]. Crit Care, 2018, 16 (4): 115-6.

[14] Shimizu K. Ogura H. et al. Communicating by electrolarynx with a blind tetraplegic spinal cord injury patient on mechanical ventilation in the ICU [J]. Spinal Cord, 2019, 51 (4): 341-2.

[15] Brummel NE. Jackson JC. et al. A combined early cognitive and physical rehabilitation program for people who are critically ill: the activity and cognitive therapy in the intensive care unit (ACT-ICU) trial [J]. Phys Ther, 2018, 92 (12): 1580-92.

[16] Berti JS. Tonon E. et al. Manual hyperinflation combined with expiratory rib cage compression for reduction of length of ICU stay in critically ill patients on mechanical ventilation $[\mathrm{J}]$. $\mathrm{J}$ Bras Pneumol, 2018, 38 (4): 477-86.

[17] Stevens V. Lodise TP. et al. The utility of acute physiology and chronic health evaluation II scores for prediction of mortality among intensive care unit (ICU) and non-ICU patients with methicillin-resistant Staphylococcus aureus bacteremia [J]. Infect Control Hosp Epidemiol, 2018, 33 (6): 558-64.

[18] Baudry T. Ader F. et al. Non-invasive mechanical ventilation to prevent ICU-acquired infection [J]. Infect Disord Drug Targets, 2011, 11 (4): 384-8.

[19] Rosenthal M, Gabrielli A, Moore F. The evolution of nutritional support in long term ICU patients: from multisystem organ failure to persistent inflammation immunosuppression catabolism syndrome. Minerva anestesiologica. 2016; 82 (1): 84-96. Epub 2015/02/24.

[20] Scala R. Esquinas A. Noninvasive mechanical ventilation for very old patients with limitations of care: is the ICU the most appropriate setting? [J]. Crit Care, 2018, 16 (3): 429-31.

[21] Boogaard M. Pickkers P. et al. Development and validation of PRE-DELIRIC (PREdiction of DELIRium in ICu patients) delirium prediction model for intensive care patients: observational multicentre study [J]. BMJ, 2018, 344 (2): 420-3.

[22] White AC. Joseph B. et al. Terminal withdrawal of mechanical ventilation at a long-term acute care hospital: comparison with a medical ICU [J]. Chest, 2009, 136 (2): 465-70.

[23] Bello G. Pennisi MA. et al. Nonthyroidal illness syndrome and prolonged mechanical ventilation in patients admitted to the ICU [J]. Chest, 2009, 135 (6): 1448-54.

[24] Dibert C. Families with loved ones on mechanical ventilation in the ICU found a way to face "living with dying" [J]. Evid Based Nurs, 2009, 12 (3): 96-8.

[25] Sihra L. Harris M. et al. Using the improving palliative care in the intensive care unit (IPAL-ICU) project to promote palliative care consultation [J]. J Pain Symptom Manage, 2011, 42 (5): 672-5.

[26] Santana Cabrera L. Rodriguez Gonzalez F. et al. Outcome of patients requiring mechanical ventilation after discharge of the ICU [J]. Med Clin (Barc), 2009, 132 (13): 525-7.

[27] Mitasova A. Kostalova M. et al. Poststroke delirium incidence and outcomes: validation of the Confusion Assessment Method for the Intensive Care Unit (CAM-ICU) [J]. Crit Care Med, 2018, 40(1): 484-90.

[28] Bambi S. Nursing clinical practice in intensive care unit (ICU) settings [J]. Dimens Crit Care Nurs, 2018, 31 (3): 212-3.

[29] Pochard F. Psychiatric issues during and after intensive care (ICU) stays [J]. Bull Acad Natl Med, 195 (2): 377-85.

[30] Loiselle CG. Gelinas C. et al. A pre-post evaluation of the Adler/Sheiner Programme (ASP): a nursing informational programme to support families and nurses in an intensive care unit (ICU) [J]. Intensive Crit Care Nurs, 2018, 28 (1): 32-40. 